\title{
Prospects for spectral CT with Medipix detectors
}

\section{Enrico Jr. Schioppa*}

Nikhef, Amsterdam, The Netherlands

E-mail: e.schioppa@nikhef.nI

\section{Jan Visser}

Nikhef, Amsterdam, The Netherlands

E-mail: janvs@nikhef.nl

\section{Els Koffeman}

Nikhef, Amsterdam, The Netherlands

E-mail: e.koffemanenikhef.nI

\begin{abstract}
In the development of X-ray Computed Tomography (CT) in medical imaging, one is working to implement spectral information. While keeping the dose level the same, or even lower, than in conventional systems, spectral CT offers the possibility to measure energy dependent features of different tissues that will allow the extraction of additional information about the patient, eventually leading to real color $\mathrm{CT}$.

Spectral CT can be achieved through the application of energy sensitive pixel detectors, such as Medipix-based semiconductor devices and by the implementation of reconstruction algorithms where the energy information is taken into account.

In this paper, we present the latest results of our work on spectral CT with Medipix detectors and specifically on detector characterization and the development of algorithms that include energy information.
\end{abstract}

Technology and Instrumentation in Particle Physics 2014,

2-6 June, 2014

Amsterdam, the Netherlands

\footnotetext{
*Speaker.
} 


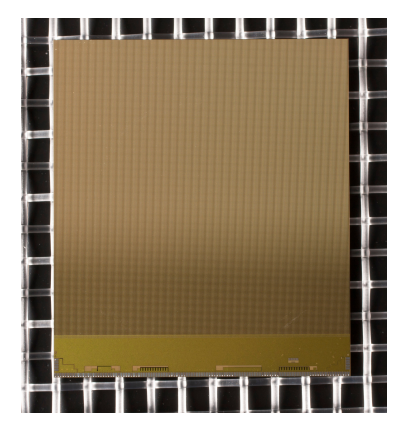

(a) Bare readout pixel matrix.

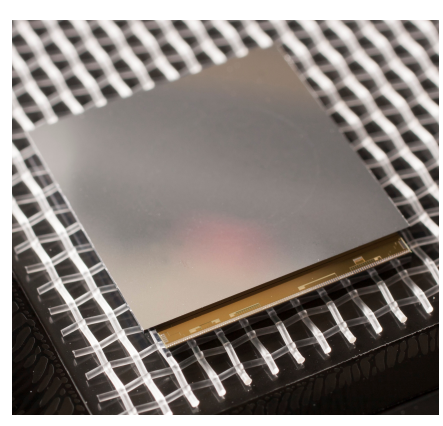

(b) ASIC bump-bonded to a silicon sensor.

Figure 1: The Medipix chip. Pictures courtesy of Jan Kopstra, Nikhef.

\section{Introduction}

Energy sensitive pixel detectors are finding applications in X-ray imaging [1, 2]. These devices not only provide a high spatial resolution, but they are also capable of energy estimation at single photon level. This means that spectral information can be added to conventional X-ray images.

Such detectors can be constructed by coupling a semiconductor pixel sensor to an energy resolving readout chip, like those of the Medipix and Timepix family [3, 4, 5]. In this case one speaks of hybrid semiconductor pixel detectors.

An important sub-field of X-ray imaging where these technologies are potentially beneficial is Computed Tomography (CT). Spectral CT can be realized by using energy sensitive pixel detectors and by implementing image reconstruction algorithms that process the spectral information contained in the X-ray data $[6,7,8]$.

In this paper, we review the main aspects of our work on spectral CT with Medipix-based devices, with focus on algorithm development and detector characterization.

\section{The Medipix chip}

Medipix chips are readout ASICs made of $55 \mu \mathrm{m}$ pitch square pixels organized on a $256 \times 256$ matrix. Connection to a semiconductor sensor can be done via bump-bonding.

The electric signal collected at the input pad of each pixel is processed by a local circuitry that performs the basic analog and digital functions. In fact, each pixel is a self-standing readout element. The signal is amplified and shaped, before being sent to a discriminator logic that compares it to a fixed threshold. Since the height of the signal is proportional to the energy lost by the particle in the sensor pixel, the threshold mechanism is in fact an energy discrimination logic.

In the Medipix 2 prototype [3], two simultaneous thresholds are available in every pixel. In this case the chip can be operated in energy window mode, where only signals falling in a specific energy range are selected. When this condition is fulfilled, a digital counter in the pixel circuitry is incremented by one unit. When, at the end of the shutter time, the chip is readout, each pixels yields a digital value telling how many quanta with energy comprised in the pre-set window were detected during the exposure.

In the Medipix3 chip [4], each pixel hosts two simultaneous counters, each connected to one of 
the discriminators. Pixels can be logically grouped in $2 \times 2$ clusters that behave as $110 \mu \mathrm{m}$ pitch super-pixels with 8 energy thresholds. Despite the loss in spacial resolution, in this situation the chip can be operated in Spectroscopic Mode (SM), where the detected X-ray quanta are binned in 8 energy channels.

\section{Image reconstruction in spectral CT}

The principle of X-ray computed tomography is to rotate the detector-source around the sample in order to collect images at different angles. These data are then fed to a dedicated algorithm that reconstructs a three dimensional image. Typically, the reconstruction is done slice by slice on sections planes orthogonal to the rotation axis.

\subsection{Beam hardening}

Different algorithms have been developed during the time, based on different approaches to the problem of tomographic reconstruction. Although most of these algorithms are derived under the assumption of monoenergetic radiation, the typical X-ray sources employed in the majority of $\mathrm{X}$-ray imaging applications are highly polychromatic. As a consequence, artifacts, known as beam hardening artifacts, may appear in the reconstructed images. In many cases, especially when the material composition of the sample is highly heterogeneous, these artifacts can significantly impair the quality of the image.

The passage from conventional CT to spectral CT is believed to have a crucial influence for the reduction or elimination of beam hardening artifacts, since the inclusion of the full spectral knowledge in the reconstruction phase makes it possible to account for this effect directly.

\subsection{Iterative reconstruction}

Beam hardening effects are a consequence of the energy dependent distortion of the source spectrum while it is transmitted through the sample. For this reason, spectral CT reconstruction algorithms must implement a model of this process.

Paradoxically, the estimation of the spectral distortion in the transmission process requires the knowledge of the inner structure of the sample, which is eventually the purpose of the reconstruction. Therefore, spectral CT algorithms must be of an iterative type, where each iteration includes an estimation of the material distribution in the object, followed by a calculation of the spectrum transmission. This approach is typical of, e.g., Expectation Maximization (EM) techniques [9]. At each iteration the object estimation improves, until the algorithm finally converges.

\subsection{Material resolution and color assignment}

The material estimation step exploits the energy information included in the data. In fact, the separation between different materials in CT reconstructions can be enhanced by analyzing the correlations among the images in the different energy channels.

When the iterative algorithm eventually converges, the resulting image is a three dimensional reconstruction ${ }^{1}$ with material resolution. For this reason, spectral CT is often referred to as material

\footnotetext{
${ }^{1}$ Or, most commonly, a stack of two dimensional reconstructions for each sample section.
} 
resolved CT. Moreover, in the data visualization phase, each material can be assigned a different color. Hence, one speaks of color CT.

\subsection{Statistical algorithms}

Another possibility for spectral CT reconstruction is to implement algorithms based on a statistical model assuming that the signal detected at each pixel is distributed according to a well defined probability density function, e.g. Poissonian. A full CT dataset can be then described in terms of a likelihood function and, according to the maximum likelihood principle, the image can be reconstructed as the one that maximizes this probability $[10,11]$.

Statistical algorithms are a promising solution for CT reconstruction, especially for their application in spectral CT, since they provide a powerful framework where energy information can be implemented naturally.

\section{The detector response}

The spectrum transmission model implemented in the spectral CT reconstruction algorithm also needs to include detector effects, mainly the absorption efficiency of the sensor material and charge sharing among pixels. Secondary effects such as scattering and fluorescence can also be included. This latter is particularly important in the case of high-Z sensor materials, such as GaAs and CdTe.

In general, these effects are energy dependent, thus giving rise to important spectral distortions during the detection process.

The detector response can be parametrized in terms of an energy response function $R\left(E, E^{\prime}\right)$, defined in such a way that, given an input spectrum $I_{i n}(E)$, the detected spectrum $I_{d e t}(E)$ can be calculated as

$$
I_{\text {det }}(E)=\int_{0}^{\infty} R\left(E, E^{\prime}\right) I_{i n}\left(E^{\prime}\right) d E^{\prime}
$$

An operative definition of the energy response function at a given energy $E^{\prime}$ is as the spectrum produced by the detector when the input is a monoenergetic spectrum $\delta\left(E-E^{\prime}\right)$. This definition provides a natural way to directly measure $R\left(E, E^{\prime}\right)$ using monochromatic sources, such as synchrotron radiation.

\subsection{Direct measurement of the energy response function using synchrotron radiation}

The most common Medipix-based X-ray detectors are produced using $300 \mu \mathrm{m}$ thick p-on-n silicon sensors. In this experiment, we aimed at measuring the energy response function of this type of sensors. A detailed description of the experiment is reported in [12].

The experiment was performed at the BM26A, the XAS (X-ray Absorption Spectroscopy) beam line of DUBBLE (Dutch-Belgian Beam Line) at the ESRF (European Synchrotron Radiation Facility, Grenoble, France) [13]. Here the detector was exposed to monochromatic beams of X-rays at different energies. The energy range spanned from $5 \mathrm{keV}$ to $32.5 \mathrm{keV}$, with steps of $2.5 \mathrm{keV}$ or less. The large beam spot allowed to illuminate several hundreds of pixels simultaneously.

The detector was operated in single threshold mode. Per-pixel cumulative spectra were collected 


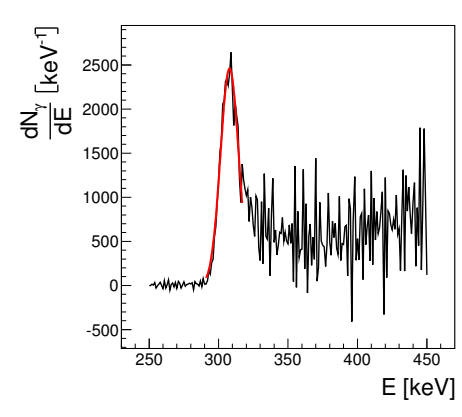

(a) Raw single-pixel spectrum.

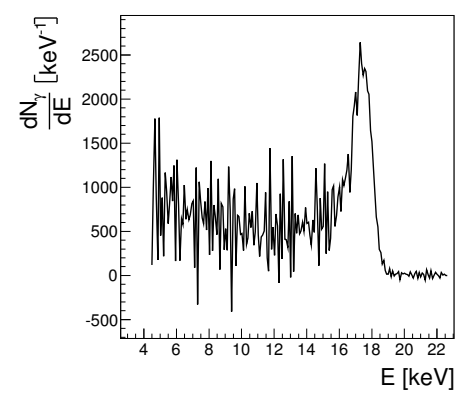

(b) Calibrated single-pixel spectrum.

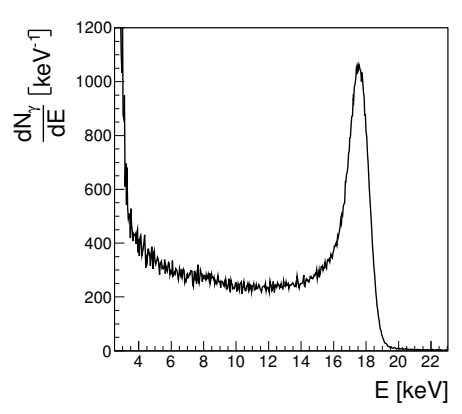

(c) Total spectrum.

Figure 2: Direct measurement of the energy response function at $17.5 \mathrm{keV}$ using synchrotron radiation.

at each beam energy by varying the threshold in fine steps. The raw spectra are then retrieved by differentiating the threshold scan, and the energy scale is calibrated.

A total spectrum at each beam energy is obtained by summing the calibrated spectra of all the pixels illuminated by the beam. Each of these spectra represents the energy response function at the corresponding beam energy. In order to fit the data and parametrize the energy response function, a numerical model is developed, based on a simple model of charge diffusion in the silicon sensor.

\subsection{Measurement of charge transport using a particle beam}

The charge diffusion properties of the detector were determined previously by a measurement with minimum ionizing particles. A single $300 \mu \mathrm{m}$ thick p-on-n silicon sensor bump bonded to four Timepix chips was taken to the H6-SPS experimental area at CERN, where it was exposed to a beam of $40 \mathrm{GeV}$ negative pions [14].

The sensor was placed longitudinally with respect to the beam. In this condition, the detector operated as a high resolution particle tracker. In fact, each particle interaction would give rise to a straight track, made of a trail of few hundred pixels each bearing the value of the energy lost by the particle in the corresponding sensor volume.

After selection, each track is fitted with a straight line. The line parameters are then used to cal- 


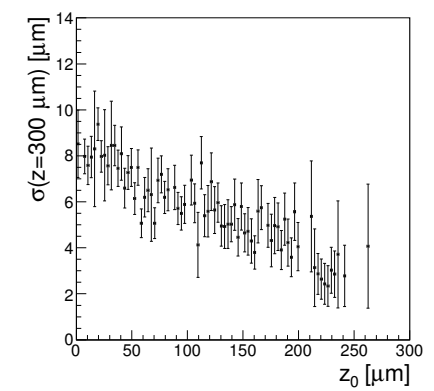

(a) The Gaussian width $\sigma$ of the charge cloud distribution as a function of the drift distance $z_{0}$.

$$
\mathrm{z}=7 \mu \mathrm{m}
$$

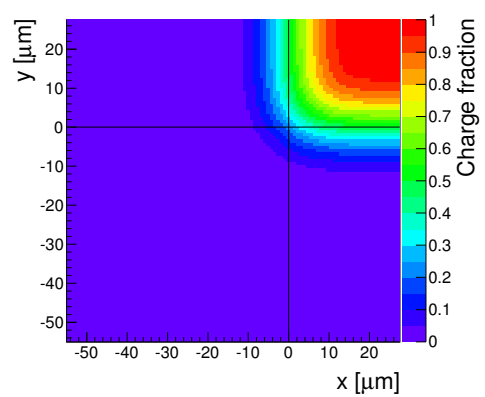

(b) Charge fraction collected by the top right pixel quarter, as a function of the position of interaction in the pixel area, at $7 \mu \mathrm{m}$ depth in the sensor.

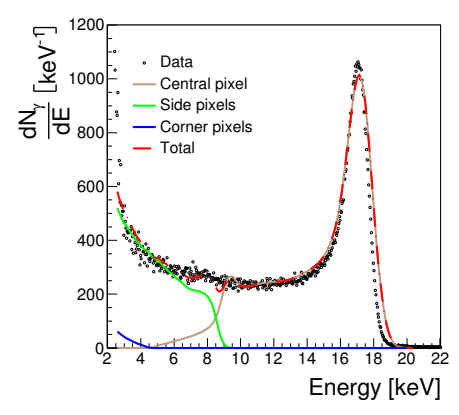

(c) The spectrum in figure $2 \mathrm{c}$ normalized and fitted with the numerical model of the detector response. Contributions from neighboring pixels are shown separately.

Figure 3: Result from the CERN test beam experiment and its application to the model of the detector response.

culate the position inside the pixel volume where the particle has interacted. This information, together with the energy information carried by each pixel in the track, allows to study the development of the cloud of charge carriers generated in the sensor as a function of the drift distance from the initiation point. This result is shown in figure $3 \mathrm{a}$.

\subsection{Numerical model of the energy response function}

Once the evolution of the charge cloud profile $\sigma\left(z, z_{0}\right)$ as a function of the drift distance $z$ for a given initiation position $\left(x_{0}, y_{0}, z_{0}\right)$ is known, the charge distribution in the pixel plane $(x, y)$ can be calculated according to a Gaussian diffusion model as 


$$
\frac{d^{2} E}{d x d y}\left(x, y ; x_{0}, y_{0}, z_{0}\right)=\frac{E^{\prime}}{\sqrt{2 \pi} \sigma\left(z, z_{0}\right)} e^{-\frac{\left(x-x_{0}\right)^{2}+\left(y-y_{0}^{2}\right)}{2 \sigma^{2}\left(z, z_{0}\right)}},
$$

where $E^{\prime}$ is the total deposited energy.

The charge that reaches the collection electrode for a given pixel is the integral in the pixel area of the charge distribution at $z=t$, where $t$ is the sensor thickness. In terms of the detected energy fraction $f=E / E^{\prime}$, this quantity takes the form

$$
f\left(x_{0}, y_{0}, z_{0}\right)=\frac{1}{\sqrt{2 \pi} \sigma\left(z, z_{0}\right)} \int_{\text {Pixel area }} e^{-\frac{\left(x-x_{0}\right)^{2}+\left(y-y_{0}^{2}\right)}{2 \sigma^{2}\left(z, z_{0}\right)}} d x d y .
$$

As an example, figure $3 b$ shows this calculation for the case when the interaction takes place at a depth of $7 \mu \mathrm{m}$ in the sensor.

The energy response function for a given input radiation spectrum $\delta\left(E-E^{\prime}\right)$ can then be calculated as

$$
R\left(E ; E^{\prime}\right)=\frac{1}{V} \int_{0}^{t} d z_{0} S_{0}\left(E ; E^{\prime}\right)\left[1-e^{-\mu(E) z_{0}}\right] \iint_{A} d x_{0} d y_{0} f\left(x_{0}, y_{0}, z_{0}\right) .
$$

In this formula $A$ is the area in the $x, y$ plane in which the surface integral is calculated, and $V$ is the corresponding volume extending through the full depth of the sensor. A doesn't simply comprise the pixel where the signal is being readout, but also the neighboring ones. This accounts for the contribution to the pixel signal from the charge cloud leaking into the neighboring pixels. Figure $3 \mathrm{c}$ shows the result for the case of $E^{\prime}=17.5 \mathrm{keV}$, where the calculated spectrum is superimposed to the measured one, previously shown in figure $2 \mathrm{c}$.

Fitting all the synchrotron spectra allows to tune the parameters of the numerical model in order to determine their dependency on energy [12]. The model can now be used to calculate the energy response function for every value of $E^{\prime}$.

Once the energy response function is known, equation 4.1 can be applied to predict the detected spectrum for any given input spectrum.

\section{Conclusion and outlook}

Providing energy sensitivity on single quanta, Medipix-based semiconductor detectors are a promising technology for applications in X-ray spectral CT.

Material resolved, color, CT can be achieved by developing dedicated reconstruction algorithms that process the spectral information provided by these devices. A crucial feature of such algorithms is that the detector response needs to be implemented.

In view of this goal, we aimed at characterizing a $300 \mu \mathrm{m}$ thick p-on-n silicon sensor readout by chips of the Medipix family. A measurement with minimum ionizing particles was performed to study the diffusion processes. The results are used to develop a numerical model for the calculation of the energy response function, whose parameters are calibrated on synchrotron data.

Current work is in progress in order to include the response function formalism into a maximum likelihood statistical reconstruction algorithm specifically designed for Medipix-based X-ray detectors, with the ultimate goal of showing the benefits these new detector technologies can contribute to the field of X-ray CT. 


\section{References}

[1] K. Taguchi and J. S. Iwanczyk, Vision 20/20: Single photon counting x-ray detectors in medical imaging, Med. Phys. 4010091 (2013) 1-19.

[2] T. Koenig et al., Imaging properties of small-pixel spectroscopic x-ray detectors based on cadmium telluride sensors, Phys. Med. Biol. 57 (2012) 6743-6759.

[3] X. Llopart et al., Medipix2: a 64-k pixel readout chip with 55- $\mu$ m square elements working in single photon counting mode, IEEE Trans. Nucl. Sci. 49 (2002) 2279-2283.

[4] R. Ballabriga et al., The Medipix3 prototype, a pixel readout chip working in single photon counting mode with improved spectrometric performance, IEEE Trans. Nucl. Sci. 54 (2007) 1824-1829.

[5] X. Llopart et al., Timepix, a 65k programmable pixel readout chip for arrival time, energy and/or photon counting measurements, Nucl. Instr. Meth. Phys. Res. A 585 (2008) 106-108 (Erratum).

[6] M. Beister et al., Iterative reconstruction methods in X-ray CT, Physica Medica 28 (2012), 94-108.

[7] M. F. Walsh et al., Spectral CT data acquisition with Medipix3.1, JINST 8 (2013), P10012.

[8] P. M. Shikhaliev and S. G. Fritz, Photon counting spectral CT versus conventional CT: comparative evaluation for breast imaging application, Phys. Med. Biol. 56 (2011) 1905-1930.

[9] L. A. Shepp and Y. Vardi, Maximum likelihood reconstruction for emission tomography, IEEE Trans. Med. Imaging M1-1(2) (1982).

[10] J. A. Fessler, Method for statistically reconstructing images from a plurality of transmission measurements having energy diversity and image reconstructor apparatus utilizing the method, US patent 6,754,298 B2, 22-06-2004.

[11] I. A. Elbakri and J. A. Fessler, Statistical Image Reconstruction for Polyenergetic X-ray Computed Tomography, IEEE Trans. Med. Imaging 21(2) (2002).

[12] E. J. Schioppa et al., Measurement of the energy response function of a silicon pixel detector readout by a Timepix chip using synchrotron radiation, JINST 9 (2014), P08002.

[13] S. Nikitenko, et al. Implementation of a combined SAXS/WAXS/QEXAFS set-up for time-resolved in situ experiments, J. Synch. Rad. 15 (2008), 632-640.

[14] E.J. Schioppa et al., Charge diffusion in semiconductor detectors using data from minimum ionizing particles, to be submitted for publication. 